\title{
Residual stress analysis of welded joints by the variational eigenstrain approach
}

\author{
Alexander M. Korsunsky ${ }^{* a}$, Gabriel Regino ${ }^{\mathrm{a}}$, David Nowell ${ }^{\mathrm{a}}$ \\ ${ }^{a}$ Department of Engineering Science, University of Oxford, Parks Road \\ Oxford OX1 3PJ, United Kingdom
}

\begin{abstract}
We present the formulation for finding the distribution of eigenstrains, i.e. the sources of residual stress, from a set of measurements of residual elastic strain (e.g. by diffraction), or residual stress, or stress redistribution, or distortion. The variational formulation employed seeks to achieve the best agreement between the model prediction and some measured parameters in the sense of a minimum of a functional given by a sum over the entire set of measurements. The advantage of this approach lies in its flexibility: different sets of measurements and information about different components of the stress-strain state can be incorporated. We demonstrate the power of the technique by analysing experimental data for welds in thin sheet of a nickel superalloy aerospace material. Very good agreement can be achieved between the prediction and the measurement results without the necessity of using iterative solution. In practice complete characterisation of residual stress states is often very difficult, due to limitations of facility access, measurement time or specimen dimensions. Implications of the new technique for experimental analysis are all the more significant, since it allows the reconstruction of the entire stress state from incomplete sets of data.
\end{abstract}

Keywords: Eigenstrain, variational calculus, residual stress, diffraction, numerical method, welds

\section{INTRODUCTION}

Reliable prediction of durability of structural components and assemblies is a fundamental requirement in various branches of engineering: transport, power generation, manufacturing and many others. This requirement led to the development of various analytical approaches to structural integrity, including elasto-plastic fracture mechanics, low cycle and high cycle fatigue analysis, creep and damage analysis, and to the introduction and standardization of appropriate methods of material property characterization. Once the material properties are determined from series of controlled laboratory experiments, numerical models of complex assemblies are used to predict the location and time of failure. Underlying this methodology is the assumption that material properties and damage accumulation models validated in the laboratory setting can be successfully transferred to the prototype (full scale object), provided certain requirements of scale independence are fulfilled.

One of the difficulties arising in the way of applying this methodology is the fact that in the process of assembly materials undergo additional processing operations that modify their internal structure (e.g. grain size and texture, composition) and internal load distribution (residual stress). Residual stress appears to be a particularly difficult parameter to account for. Unlike e.g. microstructure and composition, residual stress is associated with the entire assembly, and often undergoes significant changes if a testing piece is extracted from it for investigation. Yet residual stress is often the most crucial parameter that determines the durability of an assembled structure.

Welding is a joining and fabrication technique that relies on local melting or softening of the parent material with the purpose of allowing it to fuse together with the filler material and the opposing piece to which a joint is being made. In the process of welding the material undergoes a complex thermal and mechanical history, and the resulting assembly inherits a memory of this process in the form of microstructural and compositional changes, and residual stress distribution. In the recent decades significant efforts have been devoted by many researchers to the development of detailed numerical models of the welding process; yet at the present time reliable prediction of material structure and residual stress state at the outcome of a welding process remains elusive, its use being primarily limited to qualitative identification of, for example, the most favourable conditions for producing a weld with lower residual stress and distortion.

However, the necessity of predicting durability of assemblies in the presence of welding-induced residual stresses remains, since this joining method remains in widespread use e.g. in the aerospace industry. In this situation experimental methods of residual stress determination come to the fore, since they provide information about the central link in the chain 'processing' - 'residual stress' - 'structural integrity'. Methods of residual stress evaluation can be notionally split into 'mechanical' and 'physical'. Mechanical methods of residual stress determination rely on detecting the deformation (strain) or distortion in the test piece that arises following some cutting or material removal. Perhaps the most well-known of such techniques is hole drilling, that involves plunging a sharp fast drill into the 
surface of material, and detecting strain change from the original state at the surface of the material using a specially designed strain rosette. The results of a hole drilling experiment are interpreted using an elastic numerical model. Another method developed recently is known as the contour method [1] in which the test piece is carefully sliced using spark erosion and a two-dimensional map of displacement in the direction normal to the cutting plane is collected. This map is then used as the input for an elastic finite element numerical model of the piece, and allows the residual stress to be determined in the plane of the section.

Physical methods of residual stress evaluation rely on the determination of some parameter of the system that is known to correlate with the residual stress. Perhaps the most well-known of the physical methods of residual stress determination is X-ray diffraction. In a diffraction experiment a beam of photons is directed at a particular location within a polycrystalline sample, and an intensity profile of the scattered photons is collected, either as a function of scattering angle for fixed energy beam (monochromatic mode), or as a function of photon energy for fixed scattering angle (white beam mode). In both cases the pattern displays distinct peaks that correspond to distances between crystal lattice planes that are prevalent within the sample. If strain-free distances are known for the same sets of planes, then the measurements allow the calculation of residual direct elastic strains referring to specific orientations within the crystal and the sample.

The most widespread laboratory implementation of the X-ray diffraction method for the determination of residual stress is known as the $\sin ^{2} \psi$ technique. In this technique a series of measurements is carried out to collect the data for elastic lattice strains for a set of directions that deviate from the sample normal by a varying angle $\psi$. An assumption is then made that these measured strains correspond to a consistent strain state within homogeneous isotropic linear elastic solid, that allows the stress state within the sample to be deduced. An important observation that needs to be made at this point concerns the fact that residual stress is not measured in such an experiment, but merely deduced on the basis of certain assumptions whose validity may or may not be readily proven.

The purpose of the foregoing discussion was to establish the basic fact that residual stress state is never measured directly, be it by mechanical or physical methods, but always deduced by manipulating the results of the measurements in conjunction with certain models of deformation. It is therefore correct to say that residual stress measurement is one area of experimental activity where the development and validation of numerical models needed for the interpretation of data occupies a particularly important place: without adopting some particular model of deformation is it impossible to present measurement results in terms of residual stress.

In the present paper we are concerned with a model of residual stress generation to which we refer as the eigenstrain method. The term eigenstrain and notation $\varepsilon_{i j}^{*}$ were introduced by Toshio Mura [2] to designate any kind of permanent strain in the material arises due to some inelastic process such as plastic deformation, crystallographic transformation, thermal expansion mismatch between different parts of an assembly, etc. It is important to recognize that eigenstrains are responsible for the observed residual stresses, and therefore can be thought of as the source of residual stress; yet eigenstrain is not a priori associated with any stress, nor can it be measured directly, as it does not manifest itself in the change of crystal lattice spacing or any other physical parameter. In fact, if a uniform eigenstrain distribution is introduced into any arbitrarily shaped object, then no residual stress is produced. In other words, eigenstrains are associated with misfit and mismatch between different parts of an object. This conclusion is particularly interesting on the context of the foregoing discussion about engineering assemblies and the nature of residual stresses in them.

The following discussion is based on the analysis of the fundamental equations describing the generation of residual elastic stresses and strains from a distribution of eigenstrains. Most often the problem has to be addressed in the inverse sense: residual stresses or residual elastic strains are somehow known in a number of locations, while the unknown underlying distribution of eigenstrain sources of residual stresses needs to be found. While the direct problem of residual stress determination from eigenstrain distribution can be classed as easy (linear, elastic), the inverse problem is more difficult. However, it is important to emphasize that once the eigenstrain distribution is found, it can be used to solve the 'easy' direct problem, so that the residual stress distribution becomes known in full.

The procedure of finding the underlying eigenstrain distribution and reconstructing the complete residual stress state is entirely similar in principle to any other method of residual stress determination discussed above: the experimental data are treated using some suitable numerical model, and the residual stress state is deduced. There are some distinct advantages offered by the eigenstrain approach. Firstly, the solution obtained in this way is forced to satisfy the requirements of displacement compatibility and stress equilibrium, that often become violated if less sophisticated methods of data interpretation are used. Secondly, once the eigenstrain distribution is deduced it allows the prediction of the object's distortion and residual stress re-distribution during any subsequent machining operation, such as sectioning or surface layer removal.

In the following section we present a formulation of the direct problem of residual stress determination from the known eigenstrain distribution. We then formulate an efficient non-iterative variational approach for solving the inverse problem, and describe briefly its possible numerical implementations. We then apply the method to the analysis of experimental data for residual elastic strains in a single pass electron beam weld in a plate of aerospace 
nickel superalloy IN718, obtained using high energy synchrotron X-ray diffraction. We demonstrate how the eigenstrain distribution can be found that minimizes the disagreement between the measurements and the model prediction, and also how the method allows the refinement of the strain-free lattice parameter across the weld. Finally, we show reconstructions of the complete residual stress field within the plate.

\section{FORMULATION}

Distributions of inelastic strains contained in the sample act as sources of residual stresses. Indeed, in the absence of inelastic (permanent) strain of some origin (or indeed, when such inelastic strain is uniform throughout the sample!), then any specimen is stress free in the absence of external loading. For a known non-uniform eigenstrain distribution $\varepsilon_{i j}^{*}\left(\mathbf{x}^{\prime}\right)$, then elastic residual strains (and hence residual stresses) in the body can be found by the formulae [2]:

$$
e_{k l}(\mathbf{x})=-\varepsilon_{k l}^{*}(\mathbf{x})-\int_{-\infty}^{\infty} C_{p q m n} \varepsilon_{m n}^{*}\left(\mathbf{x}^{\prime}\right) G_{k p, q l}\left(\mathbf{x}, \mathbf{x}^{\prime}\right) \mathrm{d} \mathbf{x}^{\prime}, \quad \sigma_{i j}(\mathbf{x})=C_{i j k l} e_{k l}(\mathbf{x}) .
$$

The above formula is in principle applicable to bodies of arbitrarily complex shape, provided the elastic constants $C_{i j k l}$ are known, together with the corresponding Green's function $G_{k p}\left(\mathbf{x}, \mathbf{x}^{\prime}\right)$. In practice Green's functions can be found only for bodies of simple geometry, e.g. infinitely extended two-dimensional or three-dimensional solid. The fundamental value of the above formula, however, lies in the statement that for known eigenstrain distribution the elastic response of the body containing it can be readily found.

For convoluted geometries the finite element method provides a suitable method of solving the above direct problem of finding residual elastic strains from given eigenstrains. We are interested here in the problem that often arises in residual stress measurement and interpretation. Let there be given a set of measurements (with certain accuracy) of strains and stresses collected from a finite number of points (sampling volumes) within a bounded specimen. We would like to solve the inverse problem about the determination of unknown eigenstrains $\varepsilon_{i j}^{*}\left(\mathbf{x}^{\prime}\right)$ from this incomplete knowledge of elastic strains or residual stresses. The limited accuracy and lack of completeness of measurements suggest that direct inversion of (1) may not be the preferred solution. In fact the method chosen must be sufficiently robust to furnish approximate solutions even in this case.

We use a variational formulation for the inverse problem. Firstly, a functional of the following type (here in terms of strain) is defined [3]:

$$
J=\sum_{i=1, \ldots N} w_{i}\left[e_{k l}\left(\mathbf{x}_{i}\right)+\varepsilon_{k l}^{*}\left(\mathbf{x}_{i}\right)+\int_{-\infty}^{\infty} C_{p q m n} \varepsilon_{m n}^{*}\left(\mathbf{x}^{\prime}\right) G_{k p, q l}\left(\mathbf{x}_{i}-\mathbf{x}^{\prime}\right) \mathrm{d} \mathbf{x}^{\prime}\right]^{2} .
$$

Here $w_{i}$ denotes the weight assigned to each of the $N$ collocation points $\mathbf{x}_{i}$ considered. The first term in brackets, $e_{k l}\left(\mathbf{x}_{i}\right)$, has the meaning of the elastic strain that is observed or measured as a function of position. The remaining two terms depend on the choice of eigenstrain distribution $\varepsilon_{k l}^{*}(\mathbf{x})$, and have the meaning of the predicted elastic strain $\widetilde{e}_{k l}\left(\mathbf{x}_{i}\right)$ at a collocation point $\mathbf{x}_{i}$.

Thus, an alternative form in which equation (2) can be written is as follows:

$$
J=\sum_{i=1, \ldots N} w_{i}\left[e_{k l}\left(\mathbf{x}_{i}\right)-\tilde{e}_{k l}\left(\mathbf{x}_{i}\right)\right]^{2}
$$

Now in order to find the most likely distribution of eigenstrain $\varepsilon_{k l}^{*}(\mathbf{x})$ that gives rise to the observed elastic strain distribution, $e_{k l}(\mathbf{x})$, we require the minimum value of the functional $J$ in equation (3). This formulation corresponds to the choice of best agreement between prediction and observation in the least squares sense. In practice the step is best accomplished by expressing the unknown distribution $\varepsilon_{k l}^{*}(\mathbf{x})$ in the form of a truncated series of functions with unknown coefficients, and determining the values of these by minimising $J$ in equation (2). The choice of the weights $w_{\mathrm{i}}$ remains entirely at our disposal, and can be made judiciously (e.g. taking into account the measurement accuracy at each point $\mathbf{x}_{i}$ ) to improve the convergence.

The process of finding an approximate solution of (3) consists of the following steps: 
1) Parameterisation of the unknown eigenstrain distribution, e.g. in the form of a truncated series of polynomials, or smooth radial basis functions (RBF), wavelets, etc. with unknown vector of coefficients $a_{\mathrm{m}}$ :

$$
\tilde{e}_{k l}(\mathbf{x})=\sum_{m=1, \ldots M} a_{m} \tilde{e}_{k l}^{m}(\mathbf{x}) \text {. }
$$

2) Analytical calculation of the gradient of $J$ with respect to the parameter vector $a_{\mathrm{m}}$, leading to the linear system of equations:

$$
\frac{\mathrm{d} J}{\mathrm{~d} a_{m}}=2\left(\sum_{i=1, \ldots N} w_{i} \tilde{e}_{k l}^{m}\left(\mathbf{x}_{i}\right) \sum_{n=1, \ldots, M} a_{n} \tilde{e}_{k l}^{n}\left(\mathbf{x}_{i}\right)-\sum_{i=1, \ldots N} w_{i} \tilde{e}_{k l}^{m}\left(\mathbf{x}_{i}\right) e_{k l}\left(\mathbf{x}_{i}\right)\right)=0 .
$$

3) Solution of the above system to determine the best choice of parameters, $a^{*}{ }_{m}$, and the reconstruction of entire residual strain field due to the eigenstrain distribution corresponding to $a_{\mathrm{m}}=a_{\mathrm{m}}^{*}$ in (4).

Some important implications of the above algorithm must be pointed out:

1) Provided the parameterisation of the unknown eigenstrain field is linear, the above formulation results in a linear system (5) that can be solved without having to use iteration.

2) The formulation does not require any knowledge of complex material constitutive laws, since the solution is obtained on the basis of linear elasticity.

3) Due to the above property, the solution of the direct problem can be obtained quickly.

4) Once the most likely eigenstrain distribution is found (within the constraint of the imposed parameterisation), the complete residual stress and strain field everywhere within the body can be reconstructed.

5) Prediction for the sample distortion due to the introduction of residual stresses is also obtained as a by-product of the analysis.

6) Deformation of the sample can be predicted if subsequent material removal operations are considered, e.g. layer removal, machining, drilling, slitting, etc.

In order to illustrate the power of the developed technique we consider the example of a welded nickel superalloy plate.

\section{ANALYSIS AND DISCUSSION}
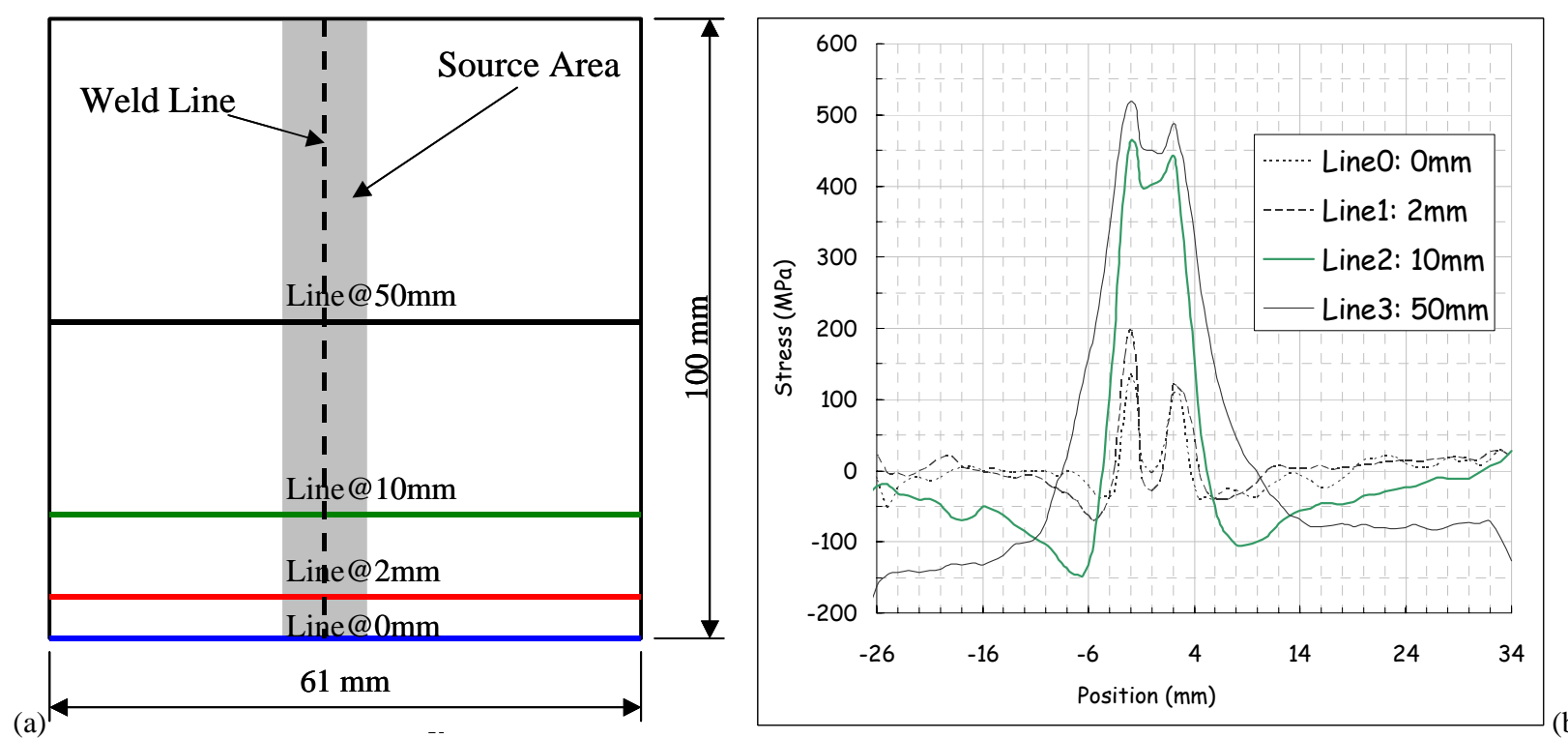

Figure 1. (a) Dimensions of the welded plate of IN718 nickel superalloy, and (b) longitudinal residual stress profiles along the lines perpendicular to the weld and lying at different distances from the plate edge. Residual stress was computed under plane stress assumptions from longitudinal and transverse residual elastic strains measured by high energy synchrotron diffraction. 
Figure 1(a) illustrates the dimensions of the experimental specimen, and the location of the measurement points. Electron beam welding was used to manufacture linear welds were produced in flat rectangular flat plates. High energy synchrotron X-ray diffraction measurements were carried out on the ID11 and ID31 beamlines at the ESRF using monochromatic mode and a scanning diffractometer. The energy of about $70 \mathrm{keV}$ was selected by the monochromator, and the 111 reflection was used. The beam spot size on the sample was approximately $1 \mathrm{~mm}$ (horizontal) by $0.25 \mathrm{~mm}$ (vertical). Line scans were performed with the scan step size of $1 \mathrm{~mm}$.

Originally the results were interpreted by assuming a constant value of $d_{0}$, the unstrained lattice spacing, everywhere within the plate. However, this led to the unphysical result of non-zero longitudinal stress existing at the bottom edge of the plate. The calculation was then repeated imposing the stress-free condition at the bottom edge, and adjusting the variation of $d_{0}$ along the horizontal $x$-axis so as to produce stress free condition at the edge.

For simplicity of analysis the only non-zero eigenstrain component was assumed to be in the longitudinal direction. The eigenstrain distribution varied in two dimensions, transversally and longitudinally to the weld line. The results of the model predictions for longitudinal stress $\sigma_{22}$ based on the most suitable eigenstrain distribution are shown in Figures 2(a), 2(b) and 2(c). Figure 2(d) depicts the variation of eigenstrain in two dimensions, indicating that the inelastic strain introduced by welding is highest in magnitude near the edge, and drops to a minimum near the centre of the plate. This finding can be explained by the increased levels of constraint experienced by the material in the middle of the plate, compared to the plate edge.
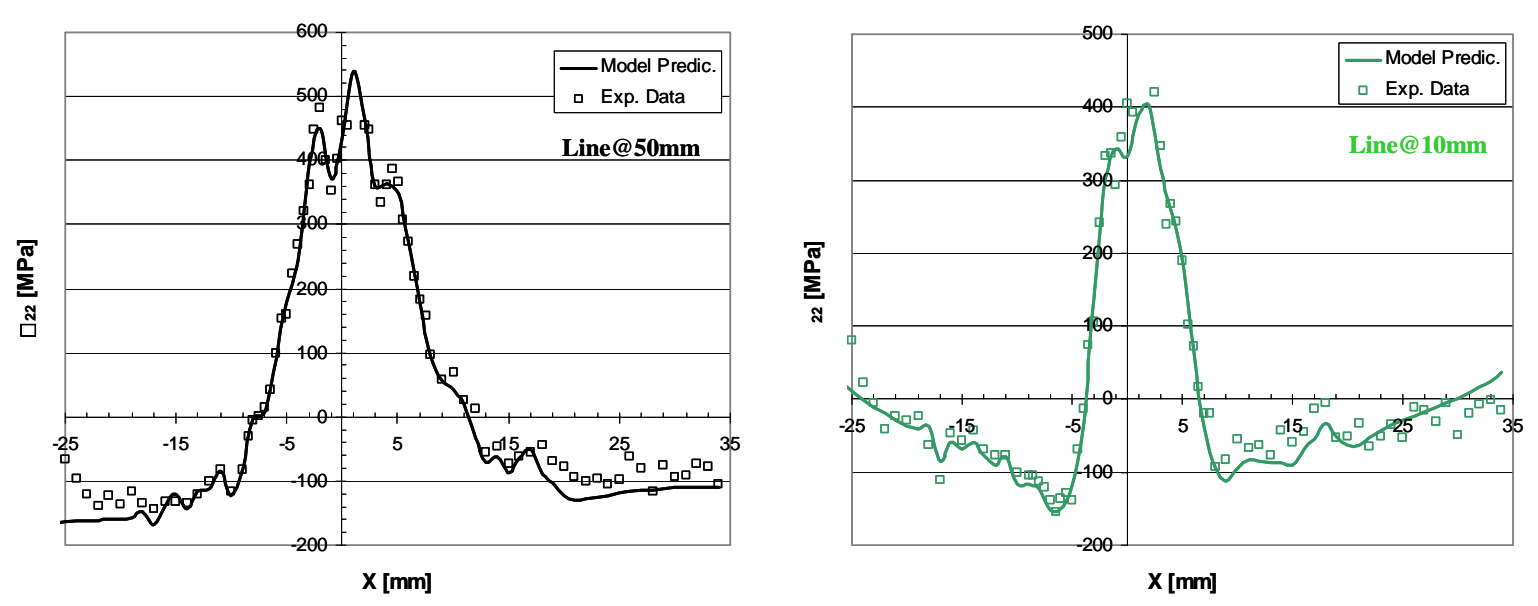

(a)

(b)
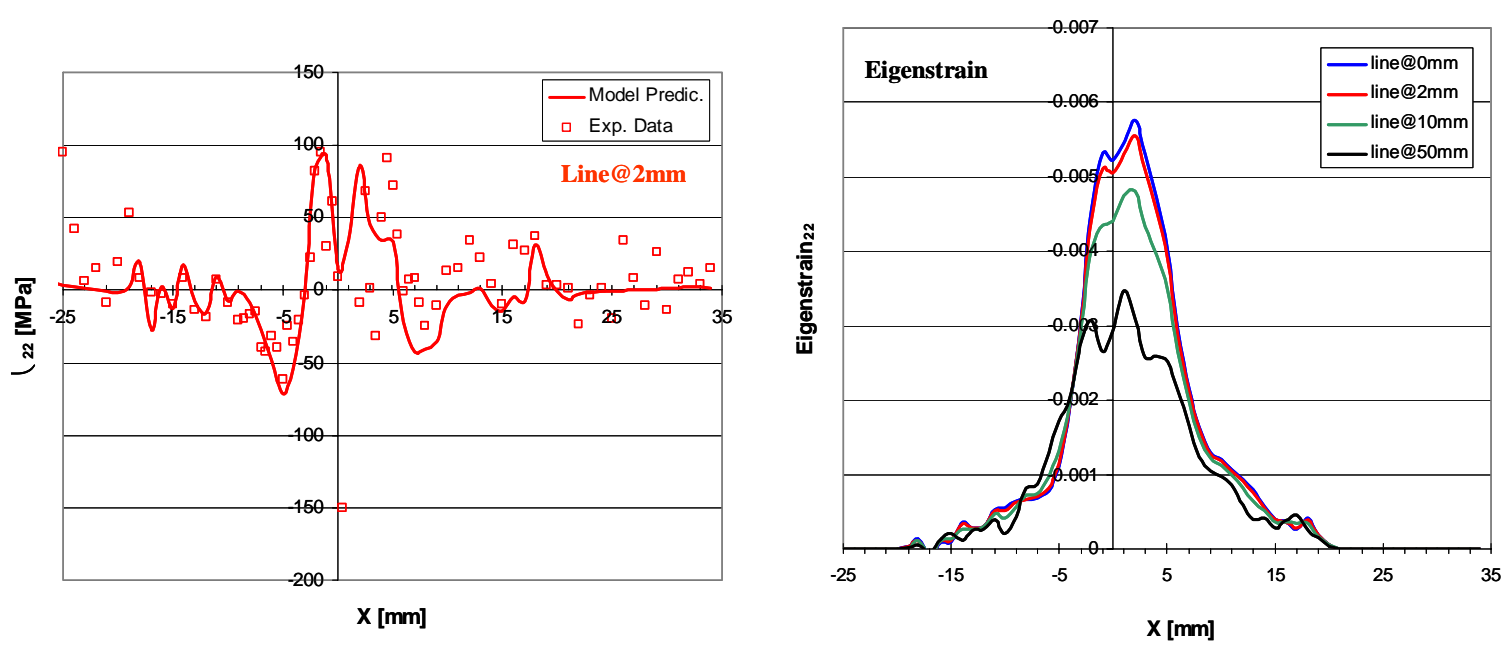

(d)

Figure 2. (a) Experimental data (markers) and eigenstrain model prediction for the longitudinal stress along the line at 50mm from the bottom edge of the plate. (b) Experimental data (markers) and eigenstrain model prediction for the longitudinal stress along the line at $10 \mathrm{~mm}$ from the bottom edge of the plate. (c) Experimental data (markers) and eigenstrain model prediction for the longitudinal stress along the line at $2 \mathrm{~mm}$ from the bottom edge of the plate. (d) Illustration of the eigenstrain variation along the four horizontal lines at 50,10, 2 and $0 \mathrm{~mm}$ from the bottom edge of the plate, respectively. 
Figure 3 shows two-dimensional plots of the von Mises stress and the longitudinal stress components within the entire plate, reconstructed using the approach described in the paper. This illustrates that a very high level of detail was extracted from the limited experimental data that served as the input to the analysis. The reason for such enhanced data interpretation is that the method of solution imposes certain additional constraints associated with continuum mechanics requirements of stress equilibrium, compatibility, etc. As a consequence, the solution found automatically satisfies the boundary conditions imposed by the problems geometry.

(a)
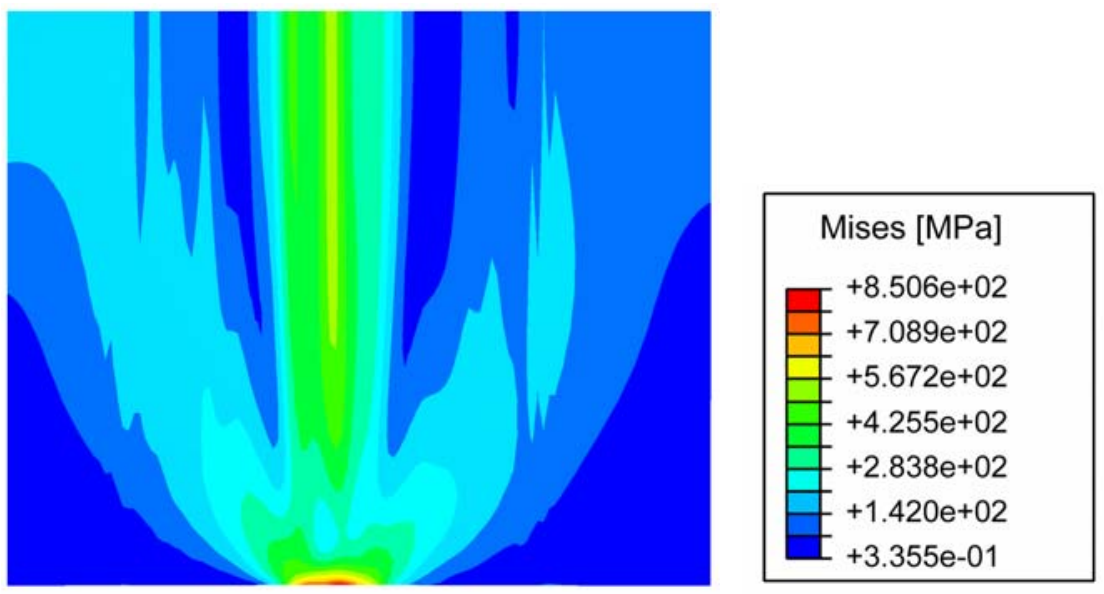

(b)

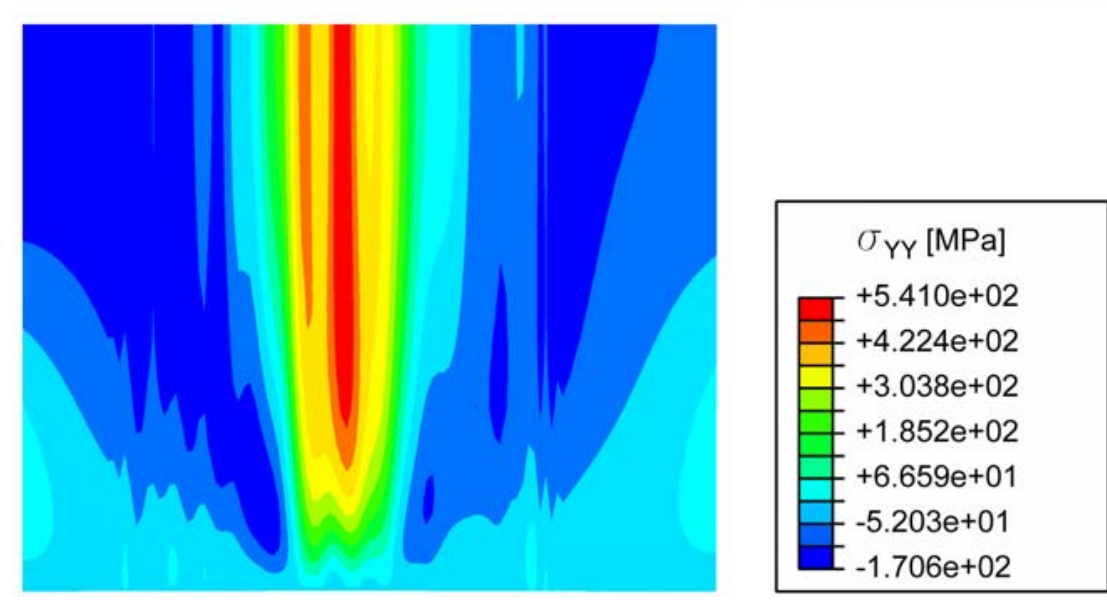

Figure 3. Reconstructed maps of (a) Von Mises and (b) longitudinal stress within the welded plate.

\section{CONCLUSION}

The present study pursued two principal aims: to develop a procedure for variational determination of the distribution of sources of residual stress (eigenstrains), and to demonstrate the applicability of the proposed approach using a practically relevant example, the interpretation of data from synchrotron-based residual stress measurements. It was shown that the inverse problem of eigenstrain distribution determination can be solved in an efficient noniterative manner, provided the parameterisation of the unknown distribution remains linear. It was also demonstrated how the proposed approach allows the reconstruction of residual stress patterns within the entire welded component based on a limited number of measurements. These results suggest that the proposed method will play an important role in the further development of residual stress analysis.

\section{REFERENCES}

1. M.B. Prime, R.J. Sebring, J.M. Edwards, D.J. Hughes, P.J. Webster, Laser Surface-Contouring and Spline Data-Smoothing for Residual Stress Measurement. Experimental Mechanics, 44(2): 176-184, 2004.

2. T. Mura, Micromechanics of Defects in Solids, Martinus Nijhoff, Dordrecht, 1987.

3. A.M. Korsunsky, G. Regino, D. Nowell, Variational determination of eigenstrain sources of residual stress. Proc. ICCES, Madeira, Portugal, 2004. 